\title{
ON THE SPACES OF $\lambda^{m}$-BOUNDED AND $\lambda^{m}$-ABSOLUTELY $p$-SUMMABLE SEQUENCES
}

\author{
Sinan Ercan and Çiğdem Asma Bektaş
}

\begin{abstract}
In this paper, we give the notion of $\lambda^{m}$-boundedness and $p$-absolute convergence of type $\lambda^{m}$ and using these notions we define new sequence spaces. We examine some topological and geometric properties of these spaces. We also establish some inclusion relations concerning these spaces and characterize some matrix classes.
\end{abstract}

Keywords: $B K$-Spaces, triangle matrix, $\alpha-, \beta-, \gamma$-duals, Schauder basis, Matrix transformations, geometric properties

\section{Introduction}

By $w$, we shall denote the space of all real or complex valued sequences. Any vector subspace of $w$ is called a sequence space. A sequence space $X$ is called an $F K$-space if it is a complete linear metric space with continuous coordinates $\tau_{k}: X \rightarrow \mathbb{C}$ defined by $\tau_{k}(x)=x_{k}$ for all $x=\left(x_{n}\right) \in X$ and every $k \in \mathbb{N}$, where $\mathbb{C}$ denotes the complex field and $\mathbb{N}=\{0,1,2, \ldots\}$. An $F K$-space whose topology is normable is called a $B K$-space. [5]

The space $\ell_{\infty}$ is $B K$-space with $\|x\|_{\infty}=\sup _{k}\left|x_{k}\right|$. Also by $\ell_{p}(0<p<\infty)$, we denote the set of $p$-absolutely convergent series. $\ell_{p}$ is a complete $p$-normed space and $B K$-space in the cases of $0<p<1$ and $1 \leq p<\infty$ with the usual $p$-norm and $\ell_{p}$-norm defined by

$$
\|x\|_{\ell_{p}}=\sum_{k=0}^{\infty}\left|x_{k}\right|^{p} ; 0<p<1
$$

and

$$
\|x\|_{\ell_{p}}=\left(\sum_{k=0}^{\infty}\left|x_{k}\right|^{p}\right)^{1 / p} ; 1 \leq p<\infty
$$

Received June 21, 2016; accepted August 06, 2016

2010 Mathematics Subject Classification. Primary 40C05; Secondary 40H05, 46A45 
respectively [24].

A sequence $\left(b_{k}\right)$ in a normed space $X$ is called a Schauder basis for $X$ if for every $x \in X$ there is a unique sequence $\left(\alpha_{k}\right)$ of scalars such that

$$
\lim _{n \rightarrow \infty}\left\|x-\sum_{k=0}^{n} \alpha_{k} b_{k}\right\|=0
$$

then $\left(b_{k}\right)$ is called Schauder basis (or briefly basis) for $X$.

Let $X, Y$ be any two sequence spaces and $A=\left(a_{n k}\right)$ be an infinite matrix of real or complex numbers $a_{n k}$, where $n, k \in \mathbb{N}$. Then $A$ defines a matrix mapping from $X$ into $Y$ and it is denoted by writing $A: X \rightarrow Y$ if for every sequence $x=\left(x_{k}\right) \in X$, the sequence $A x=\left((A x)_{n}\right)$, the $A$-transform of $x$, is in $Y$, where

$$
(A x)_{n}=\sum_{k=0}^{\infty} a_{n k} x_{k}
$$

for all $n \in \mathbb{N}$.

$(X: Y)$ denotes the class of all matrices $A$ such that $A: X \rightarrow Y$. Thus $A \in(X: Y)$ if and only if the right side of (1.1) converges for each $n \in \mathbb{N}$ and every $x \in X$, and $A x \in Y$ for all $x \in X$.

The matrix domain $X_{A}$ of an infinite matrix $A$ in a sequence space $X$ is defined by

$$
X_{A}=\{x \in w: A x \in X\}
$$

which is a sequence space.

For the sequence spaces $X, Y$ we define the set $S(X, Y)$ by

$$
S(X, Y)=\left\{z=\left(z_{k}\right) \in w: x z=\left(x_{k} z_{k}\right) \in Y \text { for all } x=\left(x_{k}\right) \in X\right\} .
$$

By the notation of (1.3), the $\alpha-, \beta-$ and $\gamma$ - duals of a sequence space $X$, which are respectively denoted by $X^{\alpha}, X^{\beta}$ and $X^{\gamma}$ are defined by

$$
X^{\alpha}=S\left(X, \ell_{1}\right), X^{\beta}=S(X, c s) \text { and } X^{\gamma}=S(X, b s) .
$$

In this paper, the collection of all nonempty and finite subsets of $\mathbb{N}$ is denoted by $\mathcal{F}$.

Definition 1.1. ([26]) A normed $X$ space is called uniformly convex if for any $\varepsilon \in(0,2]$ there exists $\delta=\delta(\varepsilon)>0$ such that if $x, y \in X$ with $\|x\|=1,\|y\|=1$ and $\|x-y\| \geq \varepsilon$, then $\left\|\frac{1}{2}(x+y)\right\| \leq 1-\delta$.

Definition 1.2. ([9]) A Banach space is super-reflexive if it is isomorphic to a Banach space that is uniformly convex.

The idea of constructing a new sequence space by means of the matrix domain of a particular limitation method has recently been employed by many authors in 
many research papers. For instance, the idea of the difference sequence spaces was introduced by Kızmaz [12] as follows,

$$
X(\Delta)=\left\{x=\left(x_{k}\right) \in w:\left(x_{k}-x_{k+1}\right) \in X\right\}
$$

where $X=\left\{\ell_{\infty}, c, c_{0}\right\}$. The space $b v_{p}$, containing sequences $\left(x_{k}\right)$ such that $\left(x_{k}-x_{k-1}\right)$ is in the sequence space $\ell_{p}$, was introduced in the case $0<p<1$ by Altay and Başar [6].

Aydın and Başar introduced the difference sequence spaces $a_{0}^{r}(\Delta)$ and $a_{c}^{r}(\Delta)$ in [8]. Polat and Altay introduced $e_{\infty}^{r}(\Delta), e_{c}^{r}(\Delta), e_{0}^{r}(\Delta)$ sequence spaces in [13]. Malkowsky and Parashar using difference operator of order $m$ studied

$$
X\left(\Delta^{(m)}\right)=\left\{x=\left(x_{k}\right) \in w: \Delta^{(m)} x \in X\right\}
$$

sequence spaces where $X=\left\{c, c_{0}, \ell_{\infty}\right\}$ in [10]. Polat and Başar defined $e_{\infty}^{r}\left(\Delta^{(m)}\right)$, $e_{c}^{r}\left(\Delta^{(m)}\right), e_{0}^{r}\left(\Delta^{(m)}\right)$ sequence spaces in [14].

Mursaleen and Noman [20] examined the notion of $\lambda$-convergence and they defined spaces of $\lambda$-bounded $\lambda$-convergent and $\lambda$-null sequences, respectively, that is,

$$
\begin{aligned}
\ell_{\infty}^{\lambda} & =\left\{x \in w: \sup _{n}\left|\Lambda_{n}(x)\right|<\infty\right\} \\
c^{\lambda} & =\left\{x \in w: \lim _{n \rightarrow \infty} \Lambda_{n}(x) \text { exists }\right\} \\
c_{0}^{\lambda} & =\left\{x \in w: \lim _{n \rightarrow \infty} \Lambda_{n}(x)=0\right\}
\end{aligned}
$$

where $\Lambda_{n}(x)=\frac{1}{\lambda_{n}} \sum_{k=0}^{n}\left(\lambda_{k}-\lambda_{k-1}\right) x_{k}$ for $n \in \mathbb{N}$. They also introduced the sequence space $\ell_{p}^{\lambda}(0<p<\infty)$ in [18] as follow;

$$
\ell_{p}^{\lambda}=\left\{x \in w: \sum_{n=0}^{\infty}\left|\Lambda_{n}(x)\right|^{p}<\infty\right\}, \quad(0<p<\infty) .
$$

Ganie and Sheikh defined sequence spaces $c\left(\Delta_{u}^{\lambda}\right)$ and $c_{0}\left(\Delta_{u}^{\lambda}\right)$ in [2]. Candan introduced $c_{0}^{\lambda}(\tilde{B})$ and $c^{\lambda}(\tilde{B})$ sequence spaces and studied some properties of these spaces in [15]. Duyar et. al. introduced $c_{0}^{\lambda}(\hat{B}), c^{\lambda}(\hat{B}), \ell_{\infty}^{\lambda}(\hat{B}), \ell_{p}^{\lambda}(\hat{B})$ sequence spaces where $1 \leq p<\infty$ in [25]. Bişgin and Sönmez defined $c_{0}^{\lambda}\left(G^{m}\right)$ and $c^{\lambda}\left(G^{m}\right)$ sequence spaces in [17]. Braha and Başar defined the $A_{\lambda}\left(\ell_{\infty}\right), A_{\lambda}(c)$ and $A_{\lambda}\left(c_{0}\right)$ sequence spaces as follows;

$$
\begin{aligned}
A_{\lambda}\left(\ell_{\infty}\right) & =\left\{x \in w: \sup _{n}\left|\left(A_{\lambda} x\right)_{n}\right|<\infty\right\}, \\
A_{\lambda}(c) & =\left\{x \in w: \exists l \in \mathbb{C} \ni \lim _{n}\left(A_{\lambda} x\right)_{n}=l\right\}, \\
A_{\lambda}\left(c_{0}\right) & =\left\{x \in w: \lim _{n}\left(A_{\lambda} x\right)_{n}=0\right\}
\end{aligned}
$$


where $\left(A_{\lambda} x\right)_{n}=\frac{1}{\Delta \lambda_{n}} \sum_{k=0}^{n}\left(\Delta^{2} \lambda_{k}\right) x_{k}$ for $n \in \mathbb{N}$ in [22].

Some other authors have constructed new sequence spaces by using the matrix domain of an infinite matrix and have studied some topological properties. (see [7], [16], [19])

\section{The notion of $\lambda^{m}$-boundedness and $p$-absolute convergence of type $\lambda^{m}$}

In this section we give the definition of $\lambda^{m}$-boundedness and $p$-absolute convergence of type $\lambda^{m}$. Let us consider the strictly increasing sequence $\lambda=\left(\lambda_{k}\right)$ of positive reals tending to infinity, that is

$$
\lim _{k \rightarrow \infty} \lambda_{k}=\infty \text { and } \lambda_{k+1} \geq m \lambda_{k}
$$

for $k, m \in \mathbb{N}$ and $m \geq 2 . \Delta^{m}$ denotes the difference operator of order $m$, that is,

$$
\Delta^{m} \lambda_{k}=\sum_{v=0}^{m}(-1)^{v}\left(\begin{array}{c}
m \\
v
\end{array}\right) \lambda_{k-v}
$$

for all $k \in \mathbb{N}$. It follows from (2.1) that $\Delta^{m} \lambda_{k} \geq 0$. We assume that any term with a negative subscript is equal to naught. e.g. $\lambda_{-1}=\lambda_{-2}=\ldots=\lambda_{-m}=0$.

We call $x=\left(x_{k}\right) \in w$ is $\lambda^{m}$-bounded if $\sup _{n}\left|\Lambda_{n}^{m}(x)\right|<\infty$, where

$$
\Lambda_{n}^{m}(x)=\frac{1}{\Delta^{m-1} \lambda_{n}} \sum_{k=0}^{n}\left(\Delta^{m} \lambda_{k}\right) x_{k}
$$

for $n \in \mathbb{N}$. Further, we say that $\sum_{k} x_{k}$ is $p$-absolutely convergent of type $\lambda^{m}$ if $\sum_{n}\left|\Lambda_{n}^{m}(x)\right|^{p}<\infty$, where $0<p<\infty$.

Now, let $x \in \ell_{\infty}$ and we have a constant $K>0$ such that $\left|x_{k}\right| \leq K$ for all $k \in \mathbb{N}$. Then, we derive that

$$
\begin{aligned}
\left|\Lambda_{n}^{m}(x)\right| & \leq \frac{1}{\Delta^{m-1} \lambda_{n}} \sum_{k=0}^{n}\left(\Delta^{m} \lambda_{k}\right)\left|x_{k}\right| \\
& \leq \frac{K}{\Delta^{m-1} \lambda_{n}} \sum_{k=0}^{n}\left(\Delta^{m} \lambda_{k}\right) \\
& =K .
\end{aligned}
$$

This means $x$ is $\lambda^{m}$-bounded. Hence we can give the following result. 
Lemma 2.1 Every bounded sequence is $\lambda^{m}$-bounded.

Lemma 2.2 If

$$
\frac{\Delta^{m-2} \lambda_{n}}{\Delta^{m-1} \lambda_{n}} \in \ell_{\infty}
$$

for $n \in \mathbb{N}$ and $m \geq 2$ then every $\lambda^{m-1}$-bounded sequence is $\lambda^{m}$-bounded.

Proof. Let take $x \in w$ is a $\lambda^{m-1}$-bounded sequence. If (2.3) holds for all $n \in \mathbb{N}$ and $m \geq 2$, then we have that

$$
\left|\Lambda_{n}^{m}(x)\right| \leq \frac{\Delta^{m-2} \lambda_{n}}{\Delta^{m-1} \lambda_{n}}\left|\Lambda_{n}^{m-1}(x)\right|+\frac{\Delta^{m-2} \lambda_{n-1}}{\Delta^{m-1} \lambda_{n}}\left|\Lambda_{n-1}^{m-1}(x)\right| .
$$

This means that $\lambda^{m-1}$-bounded sequence is also $\lambda^{m}$-bounded. As a result of this theorem we have the following property.

Remark 2.3. If (2.3) holds, every $\lambda^{r}$-bounded sequence is $\lambda^{m}$-bounded while $0<r<m$ for $m, n \in \mathbb{N}$.

3. The spaces $\ell_{p}\left(\lambda^{m}\right)(0<p<\infty)$ and $\ell_{\infty}\left(\lambda^{m}\right)$ of non-absolute type

In this section, we introduce the sequence space $\ell_{p}\left(\lambda^{m}\right)$ and $\ell_{\infty}\left(\lambda^{m}\right)$, where $0<p<\infty$. We give some topological properties. $\ell_{p}\left(\lambda^{m}\right)$ and $\ell_{\infty}\left(\lambda^{m}\right)$ sequence spaces as the of all sequences such that their $\Lambda^{m}$-transform is in the space $\ell_{p}$ and $\ell_{\infty}$, where $0<p<\infty$, i.e.,

$$
\ell_{p}\left(\lambda^{m}\right)=\left\{x=\left(x_{k}\right) \in w: \sum_{n=0}^{\infty}\left|\Lambda_{n}^{m}(x)\right|^{p}<\infty\right\}
$$

and

$$
\ell_{\infty}\left(\lambda^{m}\right)=\left\{x=\left(x_{k}\right) \in w: \sup _{n}\left|\Lambda_{n}^{m}(x)\right|<\infty\right\}
$$

where $\Lambda_{n}^{m}(x)=\frac{1}{\Delta^{m-1} \lambda_{n}} \sum_{k=0}^{n}\left(\Delta^{m} \lambda_{k}\right) x_{k}$. We can rewrite these spaces with the notation $(1.2)$

$$
\ell_{p}\left(\lambda^{m}\right)=\left(\ell_{p}\right)_{\Lambda^{m}} \text { and } \ell_{\infty}\left(\lambda^{m}\right)=\left(\ell_{\infty}\right)_{\Lambda^{m}}
$$

It is obvious by (3.1) that $\ell_{p}\left(\lambda^{m}\right)$ is a sequence space consisting of all sequences which are $p$-absolutely convergent of type $\lambda^{m}$, where $0<p<\infty$.

Now we give the following theorem with the essential in the text. 
Theorem 3.1 The following statements hold: (i) If $0<p<1$, then $\ell_{p}\left(\lambda^{m}\right)$ is a complete $p$-normed space with the $p$-norm

$$
\|x\|_{\ell_{p}\left(\lambda^{m}\right)}=\left\|\Lambda^{m}(x)\right\|_{\ell_{p}}=\sum_{n}\left|\Lambda_{n}^{m}(x)\right|^{p} ; \quad(0<p<1) .
$$

(ii) If $1 \leq p \leq \infty$, then $\ell_{p}\left(\lambda^{m}\right)$ is a $B K$-space with the norm

$$
\|x\|_{\ell_{p}\left(\lambda^{m}\right)}=\left(\sum_{n}\left|\Lambda_{n}^{m}(x)\right|^{p}\right)^{\frac{1}{p}} ; \quad(1 \leq p<\infty)
$$

and

$$
\|x\|_{\ell_{\infty}\left(\lambda^{m}\right)}=\sup _{n}\left|\Lambda_{n}^{m}(x)\right|
$$

Proof. The proof is trivial from (3.1) because of Theorem 4.3.12 in [3].

It can be easily checked that the absolute property does not hold for the space $\ell_{p}\left(\lambda^{m}\right)$, that is, $\||x|\|_{\ell_{p}\left(\lambda^{m}\right)} \neq\|x\|_{\ell_{p}\left(\lambda^{m}\right)}$ for at least one sequence $x$ in the space $\ell_{p}\left(\lambda^{m}\right)$, where $0<p \leq \infty$.

Theorem $3.2 \ell_{p}\left(\lambda^{m}\right) \cong \ell_{p}$ is isometrically isomorphic to the space $\ell_{p}$, that is $\ell_{p}\left(\lambda^{m}\right) \cong \ell_{p}$ for $0<p \leq \infty$.

Proof. Let define the transformation as $T: \ell_{p}\left(\lambda^{m}\right) \rightarrow \ell_{p}, T x=\Lambda^{m}(x) \in \ell_{p}$ for $x \in \ell_{p}\left(\lambda^{m}\right)$. The linearity of $T$ is trivial. Also, $x=0$ whenever $T x=0$ hence $T$ is injective. Now let $y=\left(y_{k}\right) \in \ell_{p}$ and define $x=\left(x_{k}\right)$ by

$$
x_{k}=\sum_{j=k-1}^{k}(-1)^{k-j} \frac{\Delta^{m-1} \lambda_{j}}{\Delta^{m} \lambda_{k}} y_{j}
$$

for $k \in \mathbb{N}$. From (2.2), we have

$$
\begin{aligned}
\Lambda_{n}^{m}(x) & =\frac{1}{\Delta^{m-1} \lambda_{n}} \sum_{k=0}^{n}\left(\Delta^{m} \lambda_{k}\right) x_{k} \\
& =\frac{1}{\Delta^{m-1} \lambda_{n}} \sum_{k=0}^{n}\left(\Delta^{m} \lambda_{k}\right)\left[\frac{\Delta^{m-1} \lambda_{k}}{\Delta^{m} \lambda_{k}} y_{k}-\frac{\Delta^{m-1} \lambda_{k-1}}{\Delta^{m} \lambda_{k}} y_{k-1}\right] \\
& =\frac{1}{\Delta \lambda_{n}} \sum_{k=0}^{n}\left[\left(\Delta^{m-1} \lambda_{k}\right) y_{k}-\left(\Delta^{m-1} \lambda_{k-1}\right) y_{k-1}\right] \\
& =y_{n}
\end{aligned}
$$

for $n \in \mathbb{N} . \Lambda^{m}(x)=y$ and since $y \in \ell_{p}$, we have $\Lambda^{m}(x) \in \ell_{p}$. Hence $x \in \ell_{p}\left(\lambda^{m}\right)$, $T x=y$ and $T$ is surjective. Also for every $x \in \ell_{p}\left(\lambda^{m}\right)$, by (3.2) and (3.3) we have

$$
\|T x\|_{\ell_{p}}=\left\|\Lambda^{m}(x)\right\|_{\ell_{p}}=\|x\|_{\ell_{p}\left(\lambda^{m}\right)} .
$$


This means $T$ is $p$-norm and norm preserving in the cases of $0<p<1$ and $1 \leq p \leq \infty$, respectively. Consequently, $T$ is isometry.

Theorem 3.3 With the exception of the case $p=2$, the space $\ell_{p}\left(\lambda^{m}\right)$ is not an inner product space, hence it is not a Hilbert space for $1 \leq p<\infty$.

Proof. We know $\ell_{2}\left(\lambda^{m}\right)$ is a $B K$-space with the norm $\|x\|_{\ell_{2}\left(\lambda^{m}\right)}=\left\|\Lambda^{m}(x)\right\|_{\ell_{2}}$. This norm can be obtained from an inner product,

$$
\|x\|_{\ell_{2}\left(\lambda^{m}\right)}=\langle x, x\rangle^{\frac{1}{2}}=\left\langle\Lambda^{m}(x), \Lambda^{m}(x)\right\rangle_{2}^{\frac{1}{2}}
$$

holds for $x \in \ell_{2}\left(\lambda^{m}\right)$. Hence $\ell_{2}\left(\lambda^{m}\right)$ is a Hilbert space. $\langle., .\rangle_{2}$ denotes the inner product on $\ell_{2}$.

Now, let us define the sequences $u=\left(u_{k}\right)$ and $v=\left(v_{k}\right)$ as follows:

$$
\begin{gathered}
u=\left(1,1,-\frac{\Delta^{m-1} \lambda_{1}}{\Delta^{m} \lambda_{2}}, 0,0, \ldots\right) \\
v=\left(1,-\frac{\Delta^{m-1}\left(\lambda_{1}+\lambda_{0}\right)}{\Delta^{m} \lambda_{1}}, \frac{\Delta^{m-1} \lambda_{1}}{\Delta^{m} \lambda_{2}}, 0,0, \ldots\right) .
\end{gathered}
$$

We have

$$
\Lambda^{m}(u)=(1,1,0,0, \ldots)
$$

and

$$
\Lambda^{m}(v)=(1,-1,0,0, \ldots) .
$$

We also have

$\|u+v\|_{\ell_{p}\left(\lambda^{m}\right)}^{2}+\|u-v\|_{\ell_{p}\left(\lambda^{m}\right)}^{2}=8 \neq 4\left(2^{2 / p}\right)=2\left(\|u\|_{\ell_{p}\left(\lambda^{m}\right)}^{2}+\|v\|_{\ell_{p}\left(\lambda^{m}\right)}^{2}\right) ; \quad(p \neq 2)$.

Hence the norm of the space $\ell_{p}\left(\lambda^{m}\right)$ does not provide the parallelogram equality. This norm cannot be obtained from an inner product. Consequently, $\ell_{p}\left(\lambda^{m}\right)$ with $p \neq 2$ is a Banach space but it is not a Hilbert space where $1 \leq p<\infty$.

We have that the domain of $X_{A}$ of an infinite triangle matrix $A=\left(a_{n k}\right)$ in a sequence space $X$ has a basis if and only if $X$ has a basis from Theorem 2.3 in [4]. Hence we have the following result:

Corollary 3.4 Let $0<p<\infty$ and define the sequence $e_{\lambda^{m}}^{(k)} \in \ell_{p}\left(\lambda^{m}\right)$ for fixed $k, n \in \mathbb{N}$ by

$$
\left(e_{\lambda^{m}}^{(k)}\right)=\left\{\begin{array}{cc}
(-1)^{n-k} \frac{\Delta^{m-1} \lambda_{k}}{\Delta^{m} \lambda_{n}} ; & k \leq n \leq k+1, \\
0 ; & \text { otherwise. }
\end{array}\right.
$$

$\left(e_{\lambda^{m}}^{(k)}\right)$ is a basis for the space $\ell_{p}\left(\lambda^{m}\right)$ and $x \in \ell_{p}\left(\lambda^{m}\right)$ has a unique representation of the form

$$
x=\sum_{k} \Lambda_{k}^{m}(x) e_{\lambda^{m}}^{(k)}
$$




\section{Some inclusion relations}

Theorem 4.1 If $0<p<q<\infty$, then the inclusion $\ell_{p}\left(\lambda^{m}\right) \subset \ell_{q}\left(\lambda^{m}\right)$ strictly holds. Proof. Let $0<p<q<\infty$. The inclusion $\ell_{p}\left(\lambda^{m}\right) \subset \ell_{q}\left(\lambda^{m}\right)$ derived by $\ell_{p} \subset \ell_{q}$. To prove that inclusion is strict we must find a sequence which is in $\ell_{q}\left(\lambda^{m}\right)$ but not in $\ell_{p}\left(\lambda^{m}\right)$. Consider the sequence $y=\left(y_{k}\right)$ in terms of the sequence $x$ for $k \in \mathbb{N}$ as follow:

$$
y_{k}=\frac{\left(\Delta^{m-1} \lambda_{k}\right) x_{k}-\left(\Delta^{m-1} \lambda_{k-1}\right) x_{k-1}}{\Delta^{m} \lambda_{k}} .
$$

We have that

$$
\begin{aligned}
\Lambda_{n}^{m}(y) & =\frac{1}{\Delta^{m-1} \lambda_{n}} \sum_{k,=0}^{n}\left[\left(\Delta^{m-1} \lambda_{k}\right) x_{k}-\left(\Delta^{m-1} \lambda_{k-1}\right) x_{k-1}\right] \\
& =x_{n}
\end{aligned}
$$

for $n \in \mathbb{N}$. This shows $\Lambda^{m}(y)=x$ and $\Lambda^{m}(y) \in \ell_{q} \backslash \ell_{p}$. Hence, the sequence $y \in \ell_{q}\left(\lambda^{m}\right)$ and $y \notin \ell_{p}\left(\lambda^{m}\right)$. The proof is completed.

Now, let $x \in w$ and $n \geq 1$. Then from (2.2) we have

$$
\begin{aligned}
x_{n}-\Lambda_{n}^{m}(x) & =\frac{1}{\Delta^{m-1} \lambda_{n}} \sum_{i=0}^{n} \Delta^{m} \lambda_{i}\left(x_{n}-x_{i}\right) \\
& =\frac{1}{\Delta^{m-1} \lambda_{n}} \sum_{i=0}^{n-1} \Delta^{m} \lambda_{i}\left(x_{n}-x_{i}\right) \\
& =\frac{1}{\Delta^{m-1} \lambda_{n}} \sum_{i=0}^{n-1}\left(\Delta^{m} \lambda_{i}\right) \sum_{k=i+1}^{n}\left(x_{k}-x_{k-1}\right) \\
& =\frac{1}{\Delta^{m-1} \lambda_{n}} \sum_{k=1}^{n}\left(x_{k}-x_{k-1}\right) \sum_{i=0}^{k-1}\left(\Delta^{m} \lambda_{i}\right) \\
& =\frac{1}{\Delta^{m-1} \lambda_{n}} \sum_{k=1}^{n} \Delta^{m-1} \lambda_{k-1}\left(x_{k}-x_{k-1}\right) .
\end{aligned}
$$

Hence we have that

$$
x_{n}-\Lambda_{n}^{m}(x)=S_{n}(x)
$$

for $n \in \mathbb{N}$. Here the sequence $S(x)=\left(S_{n}(x)\right)$ is defined by

(4.2) $\quad S_{0}(x)=0$ and $S_{n}(x)=\frac{1}{\Delta^{m-1} \lambda_{n}} \sum_{k=1}^{n}\left(\Delta^{m-1} \lambda_{k-1}\right)\left(x_{k}-x_{k-1}\right) ;(n \geq 1)$.

We have that

$$
S_{n}(x)=\frac{\Delta^{m-1} \lambda_{n-1}}{\Delta^{m} \lambda_{n}}\left[\Lambda_{n}^{m}(x)-\Lambda_{n-1}^{m}(x)\right]
$$


for $n \in \mathbb{N}$.

Theorem 4.2 The inclusion $\ell_{p}\left(\lambda^{m}\right) \subset \ell_{p}$ holds if and only if $S(x) \in \ell_{p}$ for $x \in$ $\ell_{p}\left(\lambda^{m}\right)$, where $0<p \leq \infty$.

Proof. Firstly, we suppose $\ell_{p}\left(\lambda^{m}\right) \subset \ell_{p}$ holds, where $0<p \leq \infty$. Let take any $x=\left(x_{k}\right) \in \ell_{p}\left(\lambda^{m}\right)$. By the hypothesis $x \in \ell_{p}$. From the equality given by (4.1) we have

$$
\|S(x)\|_{\ell_{p}} \leq\|x\|_{\ell_{p}}+\left\|\Lambda^{m}(x)\right\|_{\ell_{p}}=\|x\|_{\ell_{p}}+\|x\|_{\ell_{p}\left(\lambda^{m}\right)}<\infty .
$$

This shows that $S(x) \in \ell_{p}$.

Conversely, let $x \in \ell_{p}\left(\lambda^{m}\right)$ where $0<p<\infty$. By the hypothesis that $S(x) \in \ell_{p}$. By (4.1) we have that

$$
\|x\|_{\ell_{p}} \leq\|S(x)\|_{\ell_{p}}+\left\|\Lambda^{m}(x)\right\|_{\ell_{p}}=\|S(x)\|_{\ell_{p}}+\|x\|_{\ell_{p}\left(\lambda^{m}\right)}<\infty .
$$

Hence $x \in \ell_{p}$ and the inclusion $\ell_{p}\left(\lambda^{m}\right) \subset \ell_{p}$ holds.

\section{Theorem 4.3}

(i) $\ell_{p} \cap \ell_{p}\left(\lambda^{m}\right) \neq \varnothing$.

(ii) If the inclusion $\ell_{p} \subset \ell_{p}\left(\lambda^{m}\right)$ holds, then $\frac{1}{\Delta^{m-1} \lambda} \in \ell_{p}$ for $0<p<\infty$.

Proof.

(i) We can give an example. $\left(\lambda_{1}-m \lambda_{0},-\lambda_{0}, 0, \ldots\right) \in \ell_{p} \cap \ell_{p}\left(\lambda^{m}\right)$ for $0<p<\infty$.

(ii) We assume that $\ell_{p} \subset \ell_{p}\left(\lambda^{m}\right)$ holds where $0<p<\infty$. Let $x=e^{(0)}=$ $(1,0,0, \ldots) \in \ell_{p}$. Then $x \in \ell_{p}\left(\lambda^{m}\right)$ and $\Lambda^{m}(x) \in \ell_{p}$. Hence, we derive that

$$
\left(\Delta^{m} \lambda_{0}\right)^{p} \sum_{n}\left(\frac{1}{\Delta^{m-1} \lambda_{n}}\right)^{p}=\sum_{n}\left|\Lambda_{n}^{m}(x)\right|^{p}<\infty .
$$

This means $\frac{1}{\Delta^{m-1} \lambda} \in \ell_{p}$.

Theorem 4.4 If $\frac{\Delta^{m-2} \lambda_{n}}{\Delta^{m-1} \lambda_{n}} \in \ell_{p}$ for $n \in \mathbb{N}$ and $m \geq 2$, the inclusion $\ell_{p}\left(\lambda^{m-1}\right) \subset \ell_{p}\left(\lambda^{m}\right)$ holds, where $0<p<\infty$.

Proof. It can be seen by using Minkowski inequality, so we omitted it.

The following remark can be obtained from Theorem 4.4 and Lemma 2.2.

Remark 4.5. If $\frac{{\frac{\Delta}{m-2} \lambda_{n}}_{\Delta^{m-1} \lambda_{n}}}{{ }^{2}} \ell_{p}$, then $\ell_{p}\left(\lambda^{r}\right) \subset \ell_{p}\left(\lambda^{m}\right)$ holds while $0<r<m$ for $m, n \in \mathbb{N}$ where $0<p \leq \infty$.

$$
\text { 5. } \quad \alpha-, \beta-\text { and } \gamma-\text { dual of } \ell_{p}\left(\lambda^{m}\right)
$$

In this section, we give the theorems determining the $\alpha$-, $\beta$ - and $\gamma$-duals of the space $\ell_{p}\left(\lambda^{m}\right)$ where $0<p<\infty$. We begin the lemmas given in [21], which are needed in the proof of the theorems 5.4-5.6. 


\section{Lemma 5.1.}

(i) $A \in\left(\ell_{p}: \ell_{1}\right)$ if and only if

$$
\sup _{F \in \mathcal{F}} \sum_{n}\left|\sum_{k \in F} a_{n k}\right|^{q}<\infty
$$

where $1<p<\infty$ and $\frac{1}{p}+\frac{1}{q}=1$.

(ii) $A \in\left(\ell_{1}: \ell_{1}\right)$ if and only if

$$
\sup _{k} \sum_{n}\left|a_{n k}\right|<\infty .
$$

\section{Lemma 5.2.}

(i) $A \in\left(\ell_{p}: c\right)$ if and only if

$$
\lim _{n \rightarrow \infty} a_{n k} \text { exists }
$$

and

$$
\sup _{n} \sum_{k}\left|a_{n k}\right|^{q}<\infty
$$

for all $k \in \mathbb{N}$, where $1<p<\infty$ and $\frac{1}{p}+\frac{1}{q}=1$.

(ii) $A \in\left(\ell_{1}: c\right)$ if and only if (5.1) and

$$
\sup _{n, k}\left|a_{n k}\right|<\infty .
$$

\section{Lemma 5.3.}

(i) $A \in\left(\ell_{p}: \ell_{\infty}\right)$ if and only if (5.2), where $1<p<\infty$.

(ii) $A \in\left(\ell_{p}: \ell_{\infty}\right)$ if and only if (5.3), where $p=1$.

Theorem 5.4. Define the sets $B(q)$ and $C$

$$
\begin{gathered}
B(q)=\left\{a=\left(a_{n}\right) \in w: \sum_{k}\left|\frac{\Delta^{m-1} \lambda_{k}}{\Delta^{m} \lambda_{k}} a_{k}\right|^{q}<\infty\right\} \\
C=\left\{a=\left(a_{n}\right) \in w: \sup _{k}\left|\frac{\Delta^{m-1} \lambda_{k}}{\Delta^{m} \lambda_{k}} a_{k}\right|<\infty\right\} .
\end{gathered}
$$

Then $\left\{\ell_{p}\left(\lambda^{m}\right)\right\}^{\alpha}=B(q)$ and $\left\{\ell_{1}\left(\lambda^{m}\right)\right\}^{\alpha}=C$. 
Proof. Let $a=\left(a_{n}\right) \in w$ and $1<p<\infty$. We define the matrix $B=\left(b_{n k}\right)$ by

$$
b_{n k}=\left\{\begin{array}{cc}
(-1)^{n-k} \frac{\Delta^{m-1} \lambda_{k}}{\Delta^{m} \lambda_{n}} a_{n} ; & n-1 \leq k \leq n \\
0 ; & k<n-1 \text { or } k>n
\end{array}\right.
$$

and from (3.5) we have

$$
a_{n} x_{n}=\sum_{k=n-1}^{n}(-1)^{n-k} \frac{\Delta^{m-1} \lambda_{k}}{\Delta^{m} \lambda_{n}} a_{n} y_{k}=(B y)_{n} .
$$

Thus, we observe by (5.4) that $a x=\left(a_{n} x_{n}\right) \in \ell_{1}$ whenever $x=\left(x_{k}\right) \in \ell_{p}\left(\lambda^{m}\right)$ if and only if $B y \in \ell_{1}$ whenever $y=\left(y_{k}\right) \in \ell_{p}$. This means that $a=\left(a_{k}\right) \in\left\{\ell_{p}\left(\lambda^{m}\right)\right\}^{\alpha}$ if and only if $B \in\left(\ell_{p}: \ell_{1}\right)$. We obtain from Lemma $5.1 a \in\left\{\ell_{p}\left(\lambda^{m}\right)\right\}^{\alpha}$ if and only if

$$
\sup _{F \in \mathcal{F}} \sum_{k}\left|\sum_{n \in F} b_{n k}\right|^{q}<\infty .
$$

On the other hand, for $F \in \mathcal{F}$ we have that

$$
\sum_{n \in F} b_{n k}=\left\{\begin{array}{cl}
0 ; & k \notin F \text { and } k+1 \notin F \\
\frac{\Delta^{m-1} \lambda_{k}}{\Delta^{m} \lambda_{k}} a_{k} ; & k \in F \text { and } k+1 \notin F \\
-\frac{\Delta^{m}-1}{\Delta^{m} \lambda_{k}} a_{k+1} ; & k \notin F \text { and } k+1 \in F \\
\left(\frac{a_{k}}{\Delta^{m} \lambda_{k}}-\frac{a_{k+1}}{\Delta^{m} \lambda_{k+1}}\right)\left(\Delta^{m-1} \lambda_{k}\right) ; & k \in F \text { and } k+1 \in F
\end{array} .\right.
$$

Hence, we deduce that (5.4) holds if and only if

$$
\sum_{k}\left|\frac{\Delta^{m-1} \lambda_{k}}{\Delta^{m} \lambda_{k}}\right|^{q}<\infty
$$

This leads us to the consequence that $\left\{\ell_{p}\left(\lambda^{m}\right)\right\}^{\alpha}=B(q)$, where $1<p<\infty$.

Similarly, we have from (5.5) that $a=\left(a_{k}\right) \in\left\{\ell_{1}\left(\lambda^{m}\right)\right\}^{\alpha}$ if and only if $B \in\left(\ell_{1}: \ell_{1}\right)$ which can equivalently be written as

$$
\sup _{k} \sum_{n}\left|b_{n k}\right|<\infty .
$$

Also we have that

$$
\sum_{n}\left|b_{n k}\right|=\sum_{n=k}^{k+1}\left|\frac{\Delta^{m-1} \lambda_{k}}{\Delta^{m} \lambda_{n}} a_{n}\right|
$$

for $k \in \mathbb{N}$. Thus, we conclude that (5.6) holds if and only if

$$
\sup _{k}\left|\frac{\Delta^{m-1} \lambda_{k}}{\Delta^{m} \lambda_{k}} a_{k}\right|<\infty .
$$


This shows that $\left\{\ell_{1}\left(\lambda^{m}\right)\right\}^{\alpha}=C$ and this completes the proof.

Theorem 5.5. Define the set

$$
H(q)=\left\{a=\left(a_{k}\right) \in w: \sum_{k}\left|\bar{\Delta}\left(\frac{a_{k}}{\Delta^{m} \lambda_{k}}\right)\left(\Delta^{m-1} \lambda_{k}\right)\right|^{q}<\infty\right\}
$$

where

$$
\bar{\Delta}\left(\frac{a_{k}}{\Delta^{m} \lambda_{k}}\right)=\frac{a_{k}}{\Delta^{m} \lambda_{k}}-\frac{a_{k+1}}{\Delta^{m} \lambda_{k+1}} .
$$

Then $\left\{\ell_{p}\left(\lambda^{m}\right)\right\}^{\beta}=C \cap H(q)$ for $1<p<\infty$ and $\left\{\ell_{1}\left(\lambda^{m}\right)\right\}^{\beta}=C$.

Proof. Let us consider the equation

$$
\begin{aligned}
\sum_{k=0}^{n} a_{k} x_{k} & =\sum_{k=0}^{n} a_{k}\left(\sum_{j=k-1}^{k}(-1)^{k-j} \frac{\Delta^{m-1} \lambda_{j}}{\Delta^{m} \lambda_{k}} y_{j}\right) \\
& =\sum_{k=0}^{n-1}\left(\Delta^{m-1} \lambda_{k}\right) \bar{\Delta}\left(\frac{a_{k}}{\Delta^{m} \lambda_{k}}\right) y_{k}+\frac{\Delta^{m-1} \lambda_{n}}{\Delta^{m} \lambda_{n}} a_{n} y_{n} \\
& =(T y)_{n}
\end{aligned}
$$

where $n \in \mathbb{N}$ and $T=\left(t_{n k}\right)$ is the matrix defined by

$$
t_{n k}=\left\{\begin{array}{cc}
\bar{\Delta}\left(\frac{a_{k}}{\Delta^{m} \lambda_{k}}\right)\left(\Delta^{m-1} \lambda_{k}\right), & k<n \\
\frac{\Delta^{m-1} \lambda_{n}}{\Delta^{m} \lambda_{n}} a_{n}, & k=n \\
0, & k>n .
\end{array}\right.
$$

It can clearly be seen that the columns of the matrix $T$ belong to space $c$, since

$$
\lim _{n} t_{n k}=\bar{\Delta}\left(\frac{a_{k}}{\Delta^{m} \lambda_{k}}\right)\left(\Delta^{m-1} \lambda_{k}\right)
$$

for all $k \in \mathbb{N}$. Hence we deduce from the first equality given above in the proof with Lemma 5.2 that $a x=\left(a_{k} x_{k}\right) \in c s$ whenever $x=\left(x_{k}\right) \in \ell_{p}\left(\lambda^{m}\right)$ if and only if $T y \in c$ whenever $y=\left(y_{k}\right) \in \ell_{p}$. This yields that $a=\left(a_{k}\right) \in\left\{\ell_{p}\left(\lambda^{m}\right)\right\}^{\beta}$ if and only if $T \in\left(\ell_{p}: c\right)$, where $1 \leq p<\infty$. Firstly, we prove for $1<p<\infty$. We have from (5.2) that

$$
\sum_{k}\left|\bar{\Delta}\left(\frac{a_{k}}{\Delta^{m} \lambda_{k}}\right)\left(\Delta^{m-1} \lambda_{k}\right)\right|^{q}<\infty
$$

and

$$
\sup _{n}\left|\frac{\Delta^{m-1} \lambda_{n}}{\Delta^{m} \lambda_{n}} a_{n}\right|<\infty
$$


This leads us to the consequence that $\left\{\ell_{p}\left(\lambda^{m}\right)\right\}^{\beta}=C \cap H(q)$.

Then for $p=1$, from (5.3), we have that (5.7) and

$$
\sup _{k}\left|\bar{\Delta}\left(\frac{a_{k}}{\Delta^{m} \lambda_{k}}\right) \Delta^{m-1} \lambda_{k}\right|<\infty
$$

hold. But the condition (5.8) is redundant, since it is clearly seen from (5.7). Hence $\left\{\ell_{1}\left(\lambda^{m}\right)\right\}^{\beta}=C$.

Theorem 5.6. $\left\{\ell_{1}\left(\lambda^{m}\right)\right\}^{\gamma}=C$ and $\left\{\ell_{p}\left(\lambda^{m}\right)\right\}^{\gamma}=C \cap H(q)$, where $1<p<\infty$.

Proof. This can be proved similarly to the proof of Theorem 5.5 with Lemma 5.3 instead of Lemma 5.2.

\section{Some matrix mappings}

In this section, we give some results which characterize various matrix transformations between the sequence space $\ell_{p}\left(\lambda^{m}\right)$ and sequence spaces $\ell_{\infty}, c, c_{0}, \ell_{p}$ where $1 \leq p<\infty$. For any infinite matrix $A=\left(a_{n k}\right)$, we shall write for brevity reasons that

$$
\tilde{a}_{n k}=\left(\Delta^{m-1} \lambda_{k}\right) \bar{\Delta}\left(\frac{a_{n k}}{\Delta^{m} \lambda_{k}}\right)=\left(\Delta^{m-1} \lambda_{k}\right)\left(\frac{a_{n k}}{\Delta^{m} \lambda_{k}}-\frac{a_{n, k+1}}{\Delta^{m} \lambda_{k+1}}\right)
$$

for all $k, n \in \mathbb{N}$. Further, let $x, y \in w$ be connected by the relation $y=\Lambda^{m}(x)$. Then, we have by equality given in the proof of Theorem 5.5 that

$$
\sum_{k=0}^{l} a_{n k} x_{k}=\sum_{k=0}^{l-1} \tilde{a}_{n k} y_{k}+\frac{\Delta^{m-1} \lambda_{l}}{\Delta^{m} \lambda_{l}} a_{n l} y_{l}
$$

for $l, n \in \mathbb{N}$. Now we consider the following conditions:

$$
\begin{gathered}
\left(\frac{\Delta^{m-1} \lambda_{k}}{\Delta^{m} \lambda_{k}} a_{n k}\right)_{k=0}^{\infty} \in \ell_{\infty}, \\
\sup _{n, k}\left|\tilde{a}_{n k}\right|<\infty, \\
\sup _{n} \sum_{k}\left|\tilde{a}_{n k}\right|^{q}<\infty, \\
\lim _{n} \tilde{a}_{n k}=\alpha_{k} \text { for every } k \in \mathbb{N}, \\
\lim _{n} \tilde{a}_{n k}=0 \text { for every } k \in \mathbb{N},
\end{gathered}
$$




$$
\sup _{k} \sum_{n}\left|\tilde{a}_{n k}\right|^{p}<\infty
$$

By Theorem 5.5 with the results by Stieglitz and Tietz [21], we get the following result from the above conditions.

\section{Teorem 6.1.}

(i) $A=\left(a_{n k}\right) \in\left(\ell_{1}\left(\lambda^{m}\right), \ell_{\infty}\right)$ if and only if (6.1) and (6.2).

(ii) $A=\left(a_{n k}\right) \in\left(\ell_{p}\left(\lambda^{m}\right), \ell_{\infty}\right)$ if and only if (6.1) and (6.3), where $1<p<\infty$.

(iii) $A=\left(a_{n k}\right) \in\left(\ell_{p}\left(\lambda^{m}\right), c\right)$ if and only if (6.1), (6.3) and (6.4), where $1<p<\infty$.

(iv) $A=\left(a_{n k}\right) \in\left(\ell_{1}\left(\lambda^{m}\right), c\right)$ if and only if (6.1), (6.2) and (6.4).

(v) $A=\left(a_{n k}\right) \in\left(\ell_{1}\left(\lambda^{m}\right), c_{0}\right)$ if and only if (6.1), (6.2) and (6.5).

(vi) $A=\left(a_{n k}\right) \in\left(\ell_{p}\left(\lambda^{m}\right), c_{0}\right)$ if and only if (6.1), (6.3) and (6.5), where $1<p<\infty$.

(vii) $A=\left(a_{n k}\right) \in\left(\ell_{1}\left(\lambda^{m}\right), \ell_{p}\right)$ if and only if (6.1) and (6.6), where $1 \leq p<\infty$.

\section{Some geometric properties}

Theorem 7.1. $\ell_{1}\left(\lambda^{m}\right)$ is not uniformly convex.

Proof. Let take $x, y \in \ell_{1}\left(\lambda^{m}\right)$ and define such as

$$
x=\left(1, \frac{\lambda_{0}}{\Delta^{m-1}\left(\lambda_{0}-\lambda_{1}\right)}, 0, \ldots\right)
$$

and

$$
y=\left(0, \frac{\Delta^{m-1} \lambda_{1}}{\Delta^{m-1}\left(\lambda_{0}-\lambda_{1}\right)}, \frac{\Delta^{m-1} \lambda_{1}}{\Delta^{m} \lambda_{2}}, 0, \ldots\right) .
$$

Then $\|x\|_{\ell_{1}\left(\lambda^{m}\right)}=\|y\|_{\ell_{1}\left(\lambda^{m}\right)}=1,\|x-y\|_{\ell_{1}\left(\lambda^{m}\right)}=2>\varepsilon$ and $\left\|\frac{1}{2}(x+y)\right\|_{\ell_{1}\left(\lambda^{m}\right)}=1$. Hence $\ell_{1}\left(\lambda^{m}\right)$ is not uniformly convex.

Corollary 7.2. $\ell_{2}\left(\lambda^{m}\right)$ is uniformly convex and reflexive.

Proof. $\ell_{2}\left(\lambda^{m}\right)$ is a Hilbert space hence we have from Proposition 7.1.1 in [26] that $\ell_{2}\left(\lambda^{m}\right)$ is uniformly convex and from Theorem 5.5.1 given in [26] it is reflexive.

Corollary 7.3. $\ell_{p}\left(\lambda^{m}\right)$ is super-reflexive, where $1<p<\infty$.

Proof. We have from Theorem 3.2 that $\ell_{p}\left(\lambda^{m}\right)$ is isomorphic to $\ell_{p}$ and it is well known that $\ell_{p}$ is uniformly convex in [1], where $1<p<\infty$. Hence by Definition 1.2 we have the desired result. 


\section{R E F E R E N C E S}

1. A. J. Clarkson, Uniformly convex spaces, Trans. Amer. Math. Soc., 40 (1936), 396-414.

2. A. H. Ganie, N. A. Sheikh, On some new sequence spaces of non-absolute type and matrix transformations, J. Egyptian Math. Soc., 21 (2013) ,108-114.

3. A. Wilansky, Summability Through Functional Analysis, in: North-Holland Mathematics Studies, Elsevier Science Publishers, Amsterdam, New York, Oxford, 1984.

4. A. M. Jarrah, E. Malkowsky, BK-spaces, bases and linear operators, Rendiconti Circ. Mat. Palermo II, 52, 1990, 177-191.

5. B. Choudhary, S. Nanda, Functional Analysis with Applications, John Wiley \& Sons Inc., New Delhi, 1989.

6. B. Altay, F. Başar, The matrix domain and the fine spectrum of the difference operator $\Delta$ on the sequence space $\ell_{p},(0<p<1)$, Commun. Math. Anal., 2 (2007), no. 2, 1-11.

7. B. Altay, On the space of $p$-summable difference sequences of order $m,(1 \leq p<$ $\infty)$, Studia Sci. Math. Hungar., 43 (2006), no. 4, 387-402.

8. C. Aydin, F. BAŞAR, Some new difference sequence spaces, Appl. Math. Comput., 157 (2004), no. 3, 677-693.

9. C. R. James, Super reflexive spaces with bases, Pasific J. Math., 41:2 (1972), 409-419.

10. E. Malkowsky, S. D. Parashar, Matrix transformations in space of bounded and convergent difference sequence of order $m$, Analysis 17 (1997), no. 1, 87-97.

11. F. BAŞAR, Summability Theory and Its Applications, Bentham Science Publishers, e-books, Monograph, İstanbul-2012, ISBN: 978-1-60805-420-6.

12. H., Kizmaz, On certain sequence spaces, Can. Math. Bull. 24 (1981), no. 2, 169176.

13. H. Polat, B. Altay, On some new Euler difference sequence spaces, Southeast Asian Bull. Math. 30 (2006), no. 2, 209-220.

14. H. Polat, F. BAŞAR, Some euler spaces of difference sequences of order $m$, Acta Math. Sci. Ser. B Engl. Ed. 27 (2007), no. 2, 254-266.

15. M. Candan, Domain of the double sequential band matrix in the spaces of convergent and null sequences, Adv. Difference Equ. 2014, 2014:163, 18 pp.

16. M. CANDAN, Domain of the double sequential band matrix in the classical sequence spaces, J. Inequal. Appl. 2012, 2012:281, 15 pp.

17. M. C. BişGin, A. Sönmez, Two new sequence spaces generated by the composition of $m$ th order generalized difference matrix, J. Inequal. Appl. 2014, 2014:274, $20 \mathrm{pp}$.

18. M. Mursaleen, A. K. Noman, On some new sequence spaces of non-absolute type related to the spaces $\ell_{p}$ and $\ell_{\infty}$ I, Filomat 25 (2011), no. 2, 33-51.

19. M. Mursaleen, A. K. Noman, On some new sequence spaces of non-absolute type related to the spaces $\ell_{p}$ and $\ell_{\infty}$ II, Math. Commun., 16 (2011), no. 2, 383398. 
20. M. Mursaleen, A. K. Noman, On the spaces of $\lambda$-convergent sequences and bounded sequences, Thai J. Math. 8 (2010), no. 2, 311-329.

21. M. Stieglitz, H. Tietz, Matrix transformationen von Folgenraumen eine Ergebnisübersicht, Math. Z., 154 (1977), 1-16.

22. N. L. BRAHA, F. BAŞAR, On the domain of the triangle $A(\lambda)$ on the spaces of null, convergent and bounded sequences, Abstr. Appl. Anal. 2013, Art. ID 476363, 9 pp.

23. N. L. Braha, T. Mansour, On $\Lambda^{2}$-strong convergence of numerical sequences and Fourier series, Acta Math. Hungar. 141 (2013), no. 1-2, 113-126.

24. I. J. MAddox, Elements of Fuctional Analysis, 2nd ed., The University Press, Cambridge, 1988.

25. O. Duyar, S. Demiriz, O. Özdemir, On some new generalized difference sequence spaces of non absolute type, J. Math. 2014, Art. ID 876813, 13 pp.

26. S. Kesavan, Functional analysis, Hindustan Book Agency, New Delhi, India, 2009 .

Sinan Ercan

Faculty of Science

Department of Mathematics

23119 Elazı̆ , Turkey

sinanercan45@gmail.com

Çiğdem Asma Bektaş

Faculty of Science

Department of Mathematics

23119 Elazı $\breve{g}$, Turkey

cbektas@firat.edu.tr 